\title{
Seleção de linhagens de sorgo granífero eficientes e responsivas à aplicação de fósforo
}

\author{
Fabricio Rodrigues( ${ }^{(1)}$, Jurandir Vieira de Magalhães ${ }^{(2)}$, Claudia Teixeira Guimarães ${ }^{(2)}$, \\ Flávio Dessaune Tardin ${ }^{(2)}$ e Robert Eugene Schaffert ${ }^{(2)}$
}

\begin{abstract}
(1)Universidade Estadual de Goiás, Rodovia GO-330, Km 241, Anel Viário, CEP75780-000 Ipameri, GO, Brasil. E-mail: fabricio.rodrigues@ueg.br (2)Embrapa Milho e Sorgo, Rodovia MG-424, Km 45, CEP 35701-979 Sete Lagoas, MG, Brasil. E-mail: jurandir.magalhaes@embrapa.br, claudia.guimaraes@embrapa.br, flavio.tardin@embrapa.br, robert.schaffert@embrapa.br
\end{abstract}

Resumo - O objetivo deste trabalho foi selecionar linhagens de sorgo simultaneamente responsivas ao fósforo e com elevada eficiência produtiva quanto a esse nutriente. Foram avaliadas 36 linhagens endogâmicas, em delineamento de blocos ao acaso, com duas repetições. Os caracteres usados para avaliação da eficiência produtiva foram produtividade média e eficiências de absorção, de utilização e de uso de fósforo, com e sem adubação fosfatada. Para análise da responsividade ao nutriente, foram avaliados caracteres de produtividade relativa e de eficiências de recuperação aparente, fisiológica e agronômica. Há variabilidade genética entre as linhagens quanto às eficiências de absorção, de utilização e de uso do fósforo, e quanto à responsividade ao nutriente, o que sugere a possibilidade de produção de híbridos destinados a nichos de mercado diferentes. As linhagens mais responsivas foram P9401, BR007B, BR008B, SC414-12E e SC566, e as mais eficientes, sob baixa disponibilidade de fósforo, foram ATF40B, SC566, BR005R, CMSXS225 e BR012 (R6). As linhagens ATF40B, ATF54 (f61), ATF54 (f596), QL3 e SC566 apresentaram melhor desempenho simultâneo das diferentes eficiências avaliadas e da responsividade ao fósforo. Apenas a avaliação do caráter produtividade, sob diferentes disponibilidades de fósforo, já permite identificar linhagens eficientes e responsivas ao fósforo.

Termos para indexação: Sorghum bicolor, eficiência de absorção, estresse nutricional, índice de seleção, seleção simultânea.

\section{Selection of grain sorghum lines efficient and responsive to phosphorus application}

\begin{abstract}
The objective of this work was to select sorghum lines simultaneously responsive to phosphorus fertilization and with high productive efficiency regarding this nutrient. Thirty six sorghum inbred lines were evaluated in a randomized complete block design, with two replicates. The traits used to evaluate the productive efficiency were average yield and efficiencies of absorption, utilization, and use of phosphorus, with and without phosphorus fertilization. For the analysis of the responsivity to the nutrient, the evaluated traits were relative yield and apparent recovery, physiological, and agronomic efficiencies. The lines were genetically divergent as to the efficiencies of absorption, utilization, and use of phosphorus, and to the responsivity to the nutrient, suggesting the possibility of producing hybrids destined to different market niches. The most responsive lines were P9401, BR007B, BR008B, SC414-12E, and SC566, and the most efficient ones under low phosphorus availability were ATF40B, SC566, BR005R, CMSXS225, and BR012 (R6). The ATF40B, ATF54 (f61), ATF54 (f596), QL3, and SC566 lines showed better simultaneous performance for the different evaluated efficiencies and for the responsivity to phosphorus. The evaluation of productivity alone, under different phosphorus availabilities, already makes it possible to identify efficient and responsive lines to phosphorus.
\end{abstract}

Index terms: Sorghum bicolor, absorption efficiency, nutritional stress, selection index, simultaneous selection.

\section{Introdução}

A agricultura moderna é altamente dependente do fósforo derivado de rocha fosfática, que é um recurso não renovável cujas reservas globais poderão se esgotar dentro de 40 a 150 anos (McGill, 2012). Entretanto, é possível que ocorram problemas de abastecimento do fertilizante antes do previsto, por causa da concentração de reservas em um pequeno número de países. Dessa forma, a disponibilidade de $\mathrm{P}$ para as plantas representa fator chave para a segurança alimentar global e para a agricultura sustentável (Godfray et al., 2010; Malingreau et al., 2012). 
Uma estratégia adequada para enfrentar esse problema seria detectar e explorar a variabilidade genética, para aumentar a eficiência produtiva das plantas relacionada à nutrição fosfatada e à responsividade ao fósforo, o que possibilitaria redução nos gastos com fertilizantes, pela maior adaptação das plantas a solos com baixa disponibilidade do nutriente (Good et al., 2004).

A existência de variabilidade genética em relação à eficiência produtiva e à responsividade ao fósforo tem sido identificada em diferentes culturas, como sorgo [Sorghum bicolor (L.) Moench] por Rocha et al. (2010), feijão (Phaseolus vulgaris L.) por Oliveira et al. (2012) e milho (Zea mays L.) por DoVale et al. (2013). Essa diversidade constitui a base genética para programas de melhoramento e é decorrente de uma série de mecanismos fisiológicos, morfológicos e bioquímicos desenvolvidos por plantas submetidas a condições adversas de fertilidade do solo, especialmente sob baixa disponibilidade de fósforo.

O objetivo deste trabalho foi selecionar linhagens de sorgo simultaneamente responsivas ao fósforo e com elevada eficiência produtiva quanto a esse nutriente.

\section{Material e Métodos}

$\mathrm{O}$ experimento foi realizado entre fevereiro e julho de 2009, em Latossolo Vermelho-Escuro, nos sítios de fenotipagem de fósforo da Embrapa Milho e Sorgo, no Município de Sete Lagoas, MG, a $767 \mathrm{~m}$ de altitude. O solo apresentava $360 \mathrm{~g} \mathrm{~kg}^{-1}$ de areia, $100 \mathrm{~g} \mathrm{~kg}^{-1} \mathrm{de}$ silte e $540 \mathrm{~g} \mathrm{~kg}^{-1}$ de argila.

Foram utilizadas 36 linhagens endogâmicas $\left(\mathrm{F}_{7: 8}\right)$ e elites do programa de melhoramento de sorgo da Embrapa Milho e Sorgo, com comportamento contrastante quanto à eficiência produtiva e à responsividade ao fósforo. Parte das linhagens foi pré-classificada por Schaffert et al. (2001). Essas linhagens têm sido usadas na formação de novos híbridos e constituem um conjunto de linhagens experimentais provenientes de diferentes famílias ATF, BR, CMSXS e Tx, com diferentes capacidades de aquisição do P disponível no solo e níveis de tolerância ao alumínio tóxico.

Utilizou-se o delineamento experimental de blocos ao acaso, com duas repetições. As parcelas experimentais foram constituídas por duas linhas de $4 \mathrm{~m}$, com espaçamento entre plantas de $0,15 \mathrm{~m}$ e entre linhas de $0,45 \mathrm{~m}$, com duas linhas de bordaduras laterais, em todo o experimento, em cada sítio de fenotipagem. O preparo do solo foi realizado de forma convencional, com uma aração e duas gradagens. A adubação de semeadura foi realizada no sulco, de acordo com os resultados das análises de solo. As linhagens foram avaliadas sob dois níveis de fósforo: com ou sem adubação fosfatada padrão. Na adubação padrão foram aplicados $190 \mathrm{~kg} \mathrm{ha}^{-1}$ de superfosfato triplo (42\% de $\left.\mathrm{P}_{2} \mathrm{O}_{5}\right), 100 \mathrm{~kg} \mathrm{ha}^{-1}$ de cloreto de potássio $\left(60 \%\right.$ de $\left.\mathrm{K}_{2} \mathrm{O}\right)$ e $67 \mathrm{~kg} \mathrm{ha}^{-1}$ de ureia ( $45 \%$ de N), para alcançar $35 \mathrm{mg}$ $\mathrm{dm}^{-3}$ de P. No nível com baixa disponibilidade de P, não foi aplicado qualquer tipo de adubo fosfatado. Porém, aplicaram-se $110 \mathrm{~kg} \mathrm{ha}^{-1}$ de cloreto de potássio e $64 \mathrm{~kg} \mathrm{ha}^{-1}$ de ureia.

Foram retiradas 100 amostras de solo, em cada nível de disponibilidade de $\mathrm{P}$ - controle e estresse -, para análises químicas (Tabela 1). Em média, os solos sob os níveis padrão e de baixa disponibilidade de $\mathrm{P}$ apresentaram 30,5 e 6,2 $\mathrm{mg} \mathrm{dm}^{-3}$ de $\mathrm{P}$, respectivamente.

A eficiência produtiva das linhagens em condições de estresse nutricional e de adubação adequada foi avaliada por meio de: produtividade média, obtida pela pesagem dos grãos de cada parcela e transformada para $\mathrm{Mg} \mathrm{ha}^{-1}$; eficiência de absorção $\left(\mathrm{E}_{\mathrm{ABS}}\right)$, definida como a capacidade de o genótipo absorver o $\mathrm{P}$ disponível no solo, expressa pela razão entre a quantidade de $\mathrm{P}$ na planta e de $\mathrm{P}$ disponível no solo $\left(\mathrm{kg} \mathrm{kg}^{-1}\right)$; eficiência de utilização ( $\left.\mathrm{E}_{\mathrm{UTIL}}\right)$, que é a capacidade de o genótipo utilizar o P absorvido para produção de grãos, expressa pela razão entre a quantidade de matéria seca de grãos produzidos e de P na planta $\left(\mathrm{kg} \mathrm{kg}^{-1}\right)$; e eficiência de uso ( $\left.\mathrm{E}_{\mathrm{USO}}\right)$, definida como o produto entre as eficiências de absorção e de utilização de $\mathrm{P}$, expressa pela quantidade de matéria seca de grãos produzidos por cada quilo de $\mathrm{P}$ disponível no solo $\left(\mathrm{kg} \mathrm{kg}^{-1}\right)$.

Para a avaliação do teor de P na planta e nos grãos, foram utilizadas três plantas inteiras representativas de cada parcela, das quais foram obtidas as massas de matéria fresca; posteriormente, foram obtidas as massas de matéria fresca de panículas e grãos. Após trituradas, as plantas foram secas, pesadas e moídas, tendo-se retirado amostras para análises químicas, pelo método de espectrofotometria com azul de molibdênio.

A responsividade das linhagens à aplicação de fósforo foi avaliada a partir dos caracteres: produtividade relativa $\left(\mathrm{P}_{\mathrm{REL}}\right)$, definida como a diferença de produtividade entre os níveis controle e baixa 
disponibilidade de $\mathrm{P}$, dividida pela produtividade do controle e multiplicada por 100; eficiência fisiológica ( $\left.\mathrm{E}_{\mathrm{FISIO}}\right)$, obtida pela diferença de produtividade entre os dois níveis de disponibilidade de $\mathrm{P}$, por unidade $(\mathrm{kg})$ adicional de nutriente acumulado pelas plantas entre os níveis de alta e baixa disponibilidade $\left(\mathrm{kg} \mathrm{kg}^{-1}\right)$; eficiência de recuperação aparente $\left(\mathrm{E}_{\mathrm{REC}}\right)$, definida como a capacidade de o genótipo recuperar o nutriente aplicado ao solo, expressa pela diferença de produtividade entre os níveis, dividida pela diferença de $\mathrm{P}$ no solo entre os níveis $\left(\mathrm{kg} \mathrm{kg}^{-1}\right)$; e eficiência agronômica $\left(\mathrm{E}_{\mathrm{AGRO}}\right)$, expressa pela diferença de produtividade entre os níveis de disponibilidade de $\mathrm{P}$, dividida pela quantidade de $\mathrm{P}$ aplicada no nível controle $\left(\mathrm{kg} \mathrm{kg}^{-1}\right)$.

A classificação das linhagens quanto à eficiência e à responsividade ao $\mathrm{P}$ foi efetuada da seguinte forma: linhagens eficientes obtiveram produtividades acima da média, em ambientes com baixa disponibilidade de P; enquanto linhagens responsivas tiveram produtividade acima da média geral das linhagens em condições de adubação adequada. Posteriormente, as linhagens foram classificadas de acordo com a soma de postos, ou de "ranques" (Mulamba \& Mock, 1978), tendose avaliado os caracteres de resposta e de eficiência produtiva em relação ao $\mathrm{P}$, em condições de estresse. Assim, foi possível avaliar a possibilidade de utilização do caráter produtividade de grãos, em fases iniciais do programa de melhoramento, como indicador da ocorrência de ganho simultâneo quanto às diferentes eficiências nutricionais das linhagens de sorgo.

Inicialmente, os experimentos foram analisados separadamente, conforme o modelo estatístico: $Y_{i j}=m+g_{i}+b_{j}+\varepsilon_{(i j)}$, em que $Y_{i j}$ refere-se à linhagem i no bloco $\mathrm{j}$; $\mathrm{m}$ é a média geral; $\mathrm{g}_{\mathrm{i}}$ é o efeito da i-ésima linhagem; $b_{j}$ é o efeito do j-ésimo bloco; e $\varepsilon_{(\mathrm{ij})}$ é o erro experimental. Em seguida, foi realizada a análise de variância conjunta, em que as fontes de variação do modelo foram consideradas fixas e os erros, aleatórios, com uso do modelo estatístico: $\mathrm{Y}_{\mathrm{ijq}}=\mathrm{m}+\mathrm{g}_{\mathrm{i}}+\mathrm{a}_{\mathrm{q}}+\mathrm{b}_{\mathrm{j}(\mathrm{q})}$ $+(\mathrm{ga})_{\mathrm{iq}}+\varepsilon_{\mathrm{ij}(\mathrm{q})}$, em que $\mathrm{Y}_{\mathrm{ijq}}$ refere-se à observação da linhagem i no bloco j dentro do nível q; $m$ é a média geral; $\mathrm{g}_{\mathrm{i}}$ é o efeito da i-ésima linhagem; $\mathrm{a}_{\mathrm{q}}$ é o efeito do nível q de fósforo; $b_{\mathrm{j}(\mathrm{q})}$ é o efeito do j-ésimo bloco dentro do nível q; (ga) $)_{\text {iq }}$ o o efeito da interação linhagens i e níveis q de fósforo; e $\varepsilon_{\mathrm{ij}(q)}$ é o erro experimental. Procedeu-se, então, ao agrupamento das médias, pelo método de Scott \& Knott, a 5\% de probabilidade, tendo-se utilizado o programa computacional Genes (Cruz, 2006).

\section{Resultados e Discussão}

As linhagens apresentaram comportamentos divergentes para a maioria dos caracteres avaliados (Tabela 2), o que revela a existência de variabilidade genética entre elas e, também, alto valor do coeficiente de variação genética $\left(\mathrm{CV}_{\mathrm{g}}\right)$. $\mathrm{O}$ coeficiente de variação experimental variou de 10 a $35 \%$ e indicou boa precisão experimental (Parentoni, 2008).

Em condições de adubação adequada de $P$, as linhagens mais produtivas foram BR008B, SC566, BR007B, P9401 e ATF40B, que apresentaram produtividades aproximadamente $2 \mathrm{Mg}$ maiores que a média geral. Desse modo, essas linhagens têm potencial para serem utilizadas na formação de novos híbridos comerciais, destinados a sistemas de produção com nível tecnológico mais elevado, ou, ainda, na formação de populações para extração

Tabela 1. Atributos químicos do solo na camada de 0-20 cm de profundidade, obtidos em 100 amostras de solo, antes e após aplicação dos fertilizantes nos níveis tradicional (controle) e sem adubação (estresse) com $\mathrm{P}^{(1)}$.

\begin{tabular}{|c|c|c|c|c|c|c|c|c|c|c|c|}
\hline \multirow[t]{2}{*}{ Níveis } & $\mathrm{pH} \mathrm{em}$ & CTC & $\mathrm{H}+\mathrm{Al}$ & $\mathrm{Al}^{3+}$ & $\mathrm{Ca}^{2+}$ & $\mathrm{Mg}^{2+}$ & $\mathrm{K}^{+}$ & P (Mehlich 1) & \multirow{2}{*}{$\begin{array}{c}\mathrm{MO} \\
\left(\mathrm{g} \mathrm{kg}^{-1}\right)\end{array}$} & \multirow{2}{*}{\multicolumn{2}{|c|}{$\begin{array}{c}\mathrm{V} \\
\mathrm{-}\end{array}$}} \\
\hline & $\mathrm{H}_{2} \mathrm{O}$ & ------ & ------ & $\left(\mathrm{cmol}_{\mathrm{c}} \mathrm{dm}\right.$ & 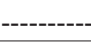 & ------- & \multicolumn{2}{|c|}{--------( $\left(\mathrm{mg} \mathrm{dm}^{-3}\right)$-------- } & & & \\
\hline & \multicolumn{11}{|c|}{ Antes da aplicação dos fertilizantes } \\
\hline Controle & $5,6 \mathrm{~b}$ & $7,3 \mathrm{mb}$ & $3,8 \mathrm{~m}$ & $0,2 \mathrm{mba}$ & $5,3 \mathrm{mb}$ & $1,2 \mathrm{~b}$ & $85 \mathrm{~b}$ & $13,3 \mathrm{~m}$ & $3,4 \mathrm{~m}$ & $64,2 b$ & $1,8 \mathrm{mba}$ \\
\hline \multirow[t]{2}{*}{ Estresse } & $5,3 b$ & $8,2 \mathrm{mb}$ & $4,8 \mathrm{~m}$ & $0,3 \mathrm{mba}$ & $2,7 \mathrm{~b}$ & $0,5 \mathrm{~m}$ & $68 \mathrm{~b}$ & $4,6 \mathrm{ba}$ & $3,4 \mathrm{~m}$ & $42,6 \mathrm{~m}$ & $10,3 \mathrm{ba}$ \\
\hline & \multicolumn{11}{|c|}{ Após a aplicação dos fertilizantes } \\
\hline Controle & $5,8 \mathrm{~b}$ & $9,1 \mathrm{mb}$ & $2,7 \mathrm{ba}$ & $0,0 \mathrm{mba}$ & $5,1 \mathrm{mb}$ & $1,1 \mathrm{~b}$ & $106,3 \mathrm{mb}$ & $30,5 \mathrm{mb}$ & $3,8 \mathrm{~m}$ & $70,5 b$ & $0,0 \mathrm{mba}$ \\
\hline Estresse & $5,5 b$ & $8,4 \mathrm{mb}$ & $4,5 \mathrm{~m}$ & $0,2 \mathrm{mba}$ & $3,1 \mathrm{~b}$ & $0,6 \mathrm{~m}$ & $75,8 \mathrm{~b}$ & $6,2 \mathrm{~m}$ & $3,5 \mathrm{~m}$ & $46,3 \mathrm{~m}$ & $2,7 \mathrm{mba}$ \\
\hline
\end{tabular}

${ }^{(1)}$ Atributos classificados segundo critérios do Sistema brasileiro de classificação de solos (1999): mb, muito bom; b, bom; m médio; ba, baixo; e mba, muito baixo. $\mathrm{MO}$, matéria orgânica do solo; $\mathrm{V}$, saturação por bases; e m, saturação por $\mathrm{Al}^{3+}$. 
de novas linhagens destinadas a esse mercado (Tabela 3).

A linhagem ATF40B foi a que apresentou maior $\mathrm{E}_{\mathrm{ABS}}$, tendo sido capaz de absorver $62 \%$ do total de $\mathrm{P}$ disponível no solo, no nível controle (Tabela 3). A fixação de fosfatos na superfície de minerais de argila, ou a precipitação com outros elementos presentes na solução do solo, tem impelido os produtores a aumentar as adubações com $\mathrm{P}_{2} \mathrm{O}_{5}$, especialmente em Latossolos, mesmo sem o auxílio de análise prévia para avaliação do P disponível no solo (Ranno et al., 2007; Santos et al., 2008). O uso de linhagens com maior $\mathrm{E}_{\mathrm{ABS}}$ poderia, assim, reduzir os custos de produção e minimizar o impacto ambiental das adubações fosfatadas (Luo et al., 2012).

Seis linhagens tiveram $\mathrm{E}_{\mathrm{UTIL}}$ acima da média geral, em condições de adubação adequada (Tabela 3 ). Essas linhagens foram agrupadas em dois grupos distintos (a e b), pelo teste de Scott \& Knott, e apresentaram $\mathrm{E}_{\text {UTIL }}$ entre 432 e $118 \mathrm{~kg} \mathrm{~kg}^{-1}$. A linhagem ATF54 (f240) apresentou $\mathrm{E}_{\mathrm{UTIL}}$ aproximadamente quatro vezes maior que a média geral das linhagens e merece estudos mais detalhados para avaliação do seu mecanismo de utilização de P. Fritsche-Neto et al. (2010) relataram que a capacidade de acúmulo de $\mathrm{P}$ pelas plantas de milho pode ser utilizada na seleção indireta de linhagens com elevada eficiência de utilização do nutriente.

$\mathrm{O}$ caráter $\mathrm{E}_{\text {Uso }}$ não discriminou bem os genótipos, e apenas dez linhagens apresentaram desempenho acima da média geral (grupo a), no nível controle (Tabela 3).
Essa variável depende de $\mathrm{E}_{\mathrm{ABS}}$ e $\mathrm{E}_{\mathrm{UTIL}}$. Portanto, o alto valor de $\mathrm{E}_{\text {Uso }}$ da linhagem BR008B (46,7 $\left.\mathrm{kg} \mathrm{kg}^{-1}\right)$ deveu-se a sua elevada capacidade de absorção de $\mathrm{P}$, enquanto o da linhagem SC566 (46,2 $\left.\mathrm{kg} \mathrm{kg}^{-1}\right)$ esteve associado a sua maior capacidade de utilização de $\mathrm{P}$.

As linhagens eficientes apresentaram produtividade média 1,42 Mg maior que a das linhagens menos eficientes (Tabela 3). Ao se considerar a proposta de classificação utilizada no presente trabalho, apenas oito linhagens poderiam ser consideradas eficientes (grupos a e b).

Em condições de baixa disponibilidade de $\mathrm{P}$, as linhagens não diferiram significativamente quanto aos caracteres $\mathrm{E}_{\mathrm{ABS}}$ e $\mathrm{E}_{\mathrm{USO}}$, indício de que apresentam as mesmas estratégias de aquisição do nutriente (Tabela 3). Entre essas estratégias, as mais comuns são o desenvolvimento de maior quantidade de raízes basais e axiais com grande número de pelos radiculares, a formação de exsudatos radiculares e o crescimento das raízes em ângulos diferentes (Ramaekers et al., 2010; Lynch, 2011).

A linhagem ATF54 (f240) também apresentou elevada $\mathrm{E}_{\text {UTIL }}$, em condição de baixa disponibilidade de $\mathrm{P}, \mathrm{e}$, portanto, teve alta capacidade de produção de grãos, com baixo acúmulo de fósforo na parte área (Tabela 3). Diferenças entre os mecanismos de absorção e utilização de $\mathrm{P}$, dentro da mesma espécie, fazem com que sejam encontradas linhagens com mecanismos fisiológicos, biológicos ou químicos mais adaptados a condições de estresse, o que já foi relatado em trigo (Triticum

Tabela 2. Estimativa dos quadrados médios das linhagens $\left(\mathrm{QM}_{\mathrm{L}}\right)$ e da interação entre linhagens e níveis de adubação $\left(\mathrm{QM}_{\mathrm{LxN}}\right)$, bem como coeficiente de variação experimental $(\mathrm{CV})$ e coeficiente de variação genética $\left(\mathrm{CV}_{\mathrm{g}}\right)$, para os parâmetros de eficiência produtiva e responsividade obtidos em condições de adubação normal (controle) e de ausência de adubação fosfatada (estresse).

\begin{tabular}{|c|c|c|c|c|c|c|c|c|}
\hline \multirow[t]{2}{*}{ Estimativa } & \multicolumn{4}{|c|}{ Eficiência produtiva $^{(1)}$} & \multicolumn{4}{|c|}{ Responsividade $^{(2)}$} \\
\hline & Produtividade & $\mathrm{E}_{\mathrm{ABS}}$ & $\mathrm{E}_{\text {UTIL }}$ & $\mathrm{E}_{\text {USO }}$ & $\mathrm{P}_{\mathrm{REL}}$ & $\mathrm{E}_{\mathrm{REC}}$ & $\mathrm{E}_{\mathrm{FISIO}}$ & $\mathrm{E}_{\mathrm{AGRO}}$ \\
\hline & \multicolumn{4}{|c|}{ Adubação controle } & \multicolumn{4}{|c|}{-} \\
\hline $\mathrm{QM}_{\mathrm{L}}$ & $3,92 * *$ & $0,02 *$ & $3.624,28 * *$ & $166,26^{*}$ & $0,05^{*}$ & $0,01 *$ & $0,22 *$ & $153,82 * *$ \\
\hline $\mathrm{CV}_{\mathrm{g}}$ & 30,17 & 29,97 & 66,89 & 21,38 & 52,50 & 27,36 & 9,12 & 71,99 \\
\hline \multirow[t]{2}{*}{ CV $(\%)$} & 9,96 & 13,46 & 11,66 & 22,40 & 19,57 & 21,30 & 19,52 & 24,04 \\
\hline & \multicolumn{4}{|c|}{ Sem adubação } & \multicolumn{4}{|c|}{-} \\
\hline $\mathrm{QM}_{\mathrm{L}}$ & $1,66 * *$ & $0,36^{\mathrm{ns}}$ & $9.481,44 * *$ & $2.945,96^{\mathrm{ns}}$ & - & - & - & - \\
\hline $\mathrm{CV}_{\mathrm{g}}$ & 25,20 & 48,27 & 48,20 & 21,81 & - & - & - & - \\
\hline \multirow[t]{2}{*}{ CV $(\%)$} & 18,39 & 35,50 & 18,51 & 31,19 & - & - & - & - \\
\hline & \multicolumn{4}{|c|}{ Análise conjunta da eficiência produtiva e da responsividade } & \multicolumn{4}{|c|}{-} \\
\hline $\mathrm{QM}_{\mathrm{L}}$ & $3,81^{\mathrm{ns}}$ & $0,68^{\text {ns }}$ & $254,43 *$ & $184,52 *$ & - & - & - & - \\
\hline $\mathrm{QM}_{\mathrm{LxN}}$ & $1,88 * *$ & $0,21^{\mathrm{ns}}$ & $3.855,96 * *$ & $175,58^{*}$ & - & - & - & - \\
\hline $\mathrm{CV}_{\mathrm{g}}$ & 22,27 & 25,78 & 62,03 & 7,44 & - & - & - & - \\
\hline CV $(\%)$ & 11,75 & 10,05 & 10,50 & 35,51 & - & - & - & - \\
\hline
\end{tabular}

${ }^{(1)} \mathrm{E}_{\mathrm{ABS}}$, eficiência de absorção; $\mathrm{E}_{\mathrm{UTIL}}$, eficiência de utilização; e $\mathrm{E}_{\mathrm{USO}}$, eficiência de uso. ${ }^{(2)} \mathrm{P}_{\mathrm{REL}}$, produtividade relativa; $\mathrm{E}_{\mathrm{REC}}$, eficiência de recuperação; $\mathrm{E}_{\mathrm{FISIO}}$, eficiência fisiológica; e $\mathrm{E}_{\mathrm{AGRO}}$, eficiência agronômica. ${ }^{\mathrm{n}} \mathrm{Não}$ significativo. ** e * Significativo pelo teste $\mathrm{F}$, a 5 e $1 \%$ de probabilidade, respectivamente. 
Tabela 3. Produtividade e classificação ${ }^{(1)}$ das linhagens de acordo com a produtividade média, e eficiências de absorção $\left(\mathrm{E}_{\mathrm{ABS}}\right)$, de utilização $\left(\mathrm{E}_{\mathrm{UTIL}}\right)$ e de uso ( $\mathrm{E}_{\mathrm{UsO}}$ ) em condições de adubação normal (controle) e ausência de adubação fosfatada (estresse), em sorgo (Sorghum bicolor) granífero ${ }^{(2)}$.

\begin{tabular}{|c|c|c|c|c|c|c|c|c|c|c|c|}
\hline \multirow{3}{*}{$\begin{array}{l}\text { Linhagem } \\
\text { ARG1 }\end{array}$} & \multicolumn{5}{|c|}{ Controle } & \multicolumn{6}{|c|}{ Estresse } \\
\hline & $\begin{array}{c}\text { Produtividade } \\
\left(\mathrm{Mg} \mathrm{ha}^{-1}\right)\end{array}$ & Classe & \multirow{2}{*}{$\begin{array}{c}\mathrm{E}_{\mathrm{ABS}} \\
0,29 \mathrm{~d}\end{array}$} & \multicolumn{2}{|c|}{$\begin{array}{c}\mathrm{E}_{\mathrm{UTIL}} \\
\mathrm{E}\end{array}$} & & $\begin{array}{l}\text { Produtividade } \\
\qquad\left(\mathrm{Mg} \mathrm{ha}^{-1}\right)\end{array}$ & Classe & \multirow{2}{*}{$\begin{array}{c}\mathrm{E}_{\mathrm{ABS}} \\
-{ }^{-}, 53 \mathrm{a}\end{array}$} & \multicolumn{2}{|c|}{ 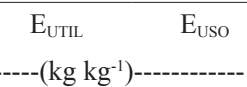 } \\
\hline & $3,51 \mathrm{c}$ & $\mathrm{R}$ & & $103,99 \mathrm{c}$ & $30,15 b$ & & $1,28 \mathrm{~d}$ & I & & $187,83 \mathrm{~d}$ & $98,83 \mathrm{a}$ \\
\hline ATF13B & $2,46 \mathrm{e}$ & NR & $0,24 \mathrm{~d}$ & $87,50 \mathrm{c}$ & $21,25 b$ & & $0,96 \mathrm{~d}$ & I & $0,17 \mathrm{a}$ & $430,08 b$ & $74,75 \mathrm{a}$ \\
\hline ATF14B & $2,30 \mathrm{e}$ & NR & $0,28 \mathrm{~d}$ & $70,34 \mathrm{~d}$ & $19,81 \mathrm{~b}$ & & $1,49 \mathrm{~d}$ & I & $0,41 \mathrm{a}$ & $281,24 \mathrm{c}$ & $115,22 \mathrm{a}$ \\
\hline ATF40B & $5,31 \mathrm{a}$ & $\mathrm{R}$ & $0,62 \mathrm{a}$ & $73,47 \mathrm{~d}$ & $45,42 \mathrm{a}$ & & $3,77 \mathrm{a}$ & $\mathrm{E}$ & $1,54 \mathrm{a}$ & $186,08 \mathrm{~d}$ & $287,35 \mathrm{a}$ \\
\hline ATF53B & $2,88 \mathrm{~d}$ & NR & $0,25 \mathrm{~d}$ & $96,93 \mathrm{c}$ & $24,65 b$ & & $0,91 \mathrm{~d}$ & I & $0,26 \mathrm{a}$ & $271,59 \mathrm{c}$ & $71,06 \mathrm{a}$ \\
\hline ATF54 (f186) & $4,10 \mathrm{c}$ & $\mathrm{R}$ & $0,34 \mathrm{c}$ & $100,48 \mathrm{c}$ & $34,52 \mathrm{a}$ & & $1,94 \mathrm{c}$ & I & $1,90 \mathrm{a}$ & $77,07 d$ & $146,30 \mathrm{a}$ \\
\hline ATF54 (f206) & $3,90 \mathrm{c}$ & $\mathrm{R}$ & $0,29 \mathrm{~d}$ & $111,53 \mathrm{c}$ & $31,95 b$ & & $1,95 \mathrm{c}$ & I & $1,04 \mathrm{a}$ & $144,10 \mathrm{~d}$ & $149,90 \mathrm{a}$ \\
\hline ATF54 (f240) & $4,50 \mathrm{~b}$ & $\mathrm{R}$ & $0,08 \mathrm{e}$ & $432,31 \mathrm{a}$ & $36,69 \mathrm{a}$ & & $1,22 \mathrm{~d}$ & I & $0,17 \mathrm{a}$ & $555,65 \mathrm{a}$ & $94,05 \mathrm{a}$ \\
\hline ATF54 (f596) & $3,97 \mathrm{c}$ & $\mathrm{R}$ & $0,44 b$ & $75,08 \mathrm{~d}$ & $33,12 \mathrm{a}$ & & $2,27 \mathrm{c}$ & I & $2,63 a$ & $66,50 \mathrm{~d}$ & $175,02 \mathrm{a}$ \\
\hline ATF54 (f61) & $3,47 \mathrm{c}$ & $\mathrm{R}$ & $0,24 \mathrm{~d}$ & $122,27 b$ & $29,52 b$ & & $2,14 \mathrm{c}$ & I & $0,53 \mathrm{a}$ & $309,94 \mathrm{c}$ & $164,90 \mathrm{a}$ \\
\hline ATF54 (f64) & $3,66 \mathrm{c}$ & $\mathrm{R}$ & $0,39 \mathrm{c}$ & $79,32 \mathrm{~d}$ & $30,58 \mathrm{~b}$ & & $1,66 \mathrm{c}$ & I & $1,27 \mathrm{a}$ & $100,43 d$ & $127,68 \mathrm{a}$ \\
\hline ATF54B & $2,92 d$ & NR & $0,17 \mathrm{e}$ & $149,28 b$ & $24,90 \mathrm{~b}$ & & $2,10 \mathrm{c}$ & I & $1,08 \mathrm{a}$ & $150,99 \mathrm{~d}$ & $163,17 \mathrm{a}$ \\
\hline BR005R & $2,89 d$ & NR & $0,22 \mathrm{~d}$ & $110,75 \mathrm{c}$ & $24,85 b$ & & $3,97 \mathrm{a}$ & E & $0,80 \mathrm{a}$ & $383,32 b$ & $305,43 a$ \\
\hline BR007B & $5,43 \mathrm{a}$ & $\mathrm{R}$ & $0,41 \mathrm{c}$ & $111,31 \mathrm{c}$ & $45,86 \mathrm{a}$ & & $1,90 \mathrm{c}$ & I & $0,91 \mathrm{a}$ & $161,87 d$ & $147,01 \mathrm{a}$ \\
\hline BR008B & $5,50 \mathrm{a}$ & $\mathrm{R}$ & $0,52 b$ & $89,76 \mathrm{c}$ & $46,78 \mathrm{a}$ & & $1,92 \mathrm{c}$ & I & $1,20 \mathrm{a}$ & $123,93 d$ & $148,64 a$ \\
\hline BR012R & $2,35 \mathrm{e}$ & NR & $0,30 \mathrm{~d}$ & $67,91 \mathrm{~d}$ & $20,25 b$ & & $1,86 \mathrm{c}$ & I & $0,88 \mathrm{a}$ & $149,65 \mathrm{~d}$ & $131,74 a$ \\
\hline BR012 (1B) & $1,78 \mathrm{f}$ & NR & $0,46 \mathrm{~b}$ & $33,36 \mathrm{~d}$ & $15,43 b$ & & $2,77 \mathrm{~b}$ & $\mathrm{E}$ & $1,28 \mathrm{a}$ & $168,28 \mathrm{~d}$ & $215,90 \mathrm{a}$ \\
\hline BR012 (2B) & $2,57 \mathrm{e}$ & NR & $0,34 \mathrm{c}$ & $65,31 \mathrm{~d}$ & $21,94 b$ & & $2,27 \mathrm{c}$ & I & $0,91 \mathrm{a}$ & $191,51 d$ & $174,58 \mathrm{a}$ \\
\hline BR012 (R6) & $2,43 \mathrm{e}$ & NR & $0,35 \mathrm{c}$ & $59,01 \mathrm{~d}$ & $20,69 b$ & & $2,90 \mathrm{~b}$ & E & $0,80 \mathrm{a}$ & $275,21 \mathrm{c}$ & $221,37 \mathrm{a}$ \\
\hline BTX623 & $2,44 \mathrm{e}$ & NR & $0,29 d$ & $71,25 \mathrm{~d}$ & $20,99 b$ & & $1,62 d$ & I & $0,88 \mathrm{a}$ & $143,45 d$ & $125,73 \mathrm{a}$ \\
\hline CMSXS110 & $3,13 d$ & NR & $0,37 \mathrm{c}$ & $72,86 \mathrm{~d}$ & $26,81 \mathrm{~b}$ & & $2,08 \mathrm{c}$ & I & $0,99 \mathrm{a}$ & $162,38 \mathrm{~d}$ & $160,65 \mathrm{a}$ \\
\hline CMSXS136 & $3,30 \mathrm{~d}$ & NR & $0,26 \mathrm{~d}$ & $106,64 \mathrm{c}$ & $28,15 b$ & & $2,33 \mathrm{c}$ & I & $1,42 \mathrm{a}$ & $114,11 \mathrm{~d}$ & $161,88 \mathrm{a}$ \\
\hline CMSXS180R & $3,87 \mathrm{c}$ & $\mathrm{R}$ & $0,34 \mathrm{c}$ & $93,01 \mathrm{c}$ & $31,17 b$ & & $2,76 b$ & E & $1,22 \mathrm{a}$ & $169,62 d$ & $206,71 \mathrm{a}$ \\
\hline CMSXS225 & $3,06 \mathrm{~d}$ & NR & $0,40 \mathrm{c}$ & $63,53 \mathrm{~d}$ & $25,32 \mathrm{~b}$ & & $2,95 \mathrm{~b}$ & E & $0,99 \mathrm{a}$ & $221,77 \mathrm{c}$ & $219,50 \mathrm{a}$ \\
\hline CMSXS226 & $3,13 \mathrm{~d}$ & NR & $0,28 \mathrm{~d}$ & $95,92 \mathrm{c}$ & $26,68 \mathrm{~b}$ & & $2,15 \mathrm{c}$ & I & $1,12 \mathrm{a}$ & $141,64 d$ & $158,77 \mathrm{a}$ \\
\hline CMSXS227 & $1,83 \mathrm{f}$ & NR & $0,23 \mathrm{~d}$ & $69,06 \mathrm{~d}$ & $15,56 \mathrm{~b}$ & & $1,41 \mathrm{~d}$ & I & $0,74 \mathrm{a}$ & $142,70 \mathrm{~d}$ & $106,03 \mathrm{a}$ \\
\hline IS3620C & $2,09 \mathrm{e}$ & NR & $0,21 \mathrm{~d}$ & $83,53 \mathrm{c}$ & $17,84 b$ & & $1,39 d$ & I & $0,62 \mathrm{a}$ & $172,89 \mathrm{~d}$ & $107,60 \mathrm{a}$ \\
\hline P9401 & $5,39 a$ & $\mathrm{R}$ & $0,47 \mathrm{~b}$ & $97,78 \mathrm{c}$ & $45,63 \mathrm{a}$ & & $1,16 \mathrm{~d}$ & I & $0,43 \mathrm{a}$ & $209,77 \mathrm{c}$ & $89,91 \mathrm{a}$ \\
\hline P9405 & $3,42 d$ & $\mathrm{R}$ & $0,51 b$ & $56,42 \mathrm{~d}$ & $28,87 \mathrm{~b}$ & & $2,69 b$ & E & $1,03 \mathrm{a}$ & $199,79 d$ & $205,55 \mathrm{a}$ \\
\hline QL3 & $4,49 b$ & $\mathrm{R}$ & $0,42 \mathrm{c}$ & $88,90 \mathrm{c}$ & $37,56 a$ & & $2,13 \mathrm{c}$ & I & $1,05 \mathrm{a}$ & $154,50 \mathrm{~d}$ & $161,86 a$ \\
\hline SC414-12E & $4,71 \mathrm{~b}$ & $\mathrm{R}$ & $0,34 \mathrm{c}$ & $117,81 \mathrm{~b}$ & $39,76 \mathrm{a}$ & & $1,88 \mathrm{c}$ & I & $0,79 \mathrm{a}$ & $184,45 \mathrm{~d}$ & $145,30 \mathrm{a}$ \\
\hline SC566 & $5,45 \mathrm{a}$ & $\mathrm{R}$ & $0,36 \mathrm{c}$ & $129,05 \mathrm{~b}$ & $46,22 \mathrm{a}$ & & $3,27 b$ & E & $1,27 \mathrm{a}$ & $199,18 \mathrm{~d}$ & $253,33 a$ \\
\hline Tx643 B & $1,52 \mathrm{f}$ & NR & $0,15 \mathrm{e}$ & $84,55 \mathrm{c}$ & $12,87 \mathrm{~b}$ & & $1,40 \mathrm{~d}$ & I & $0,67 \mathrm{a}$ & $151,50 \mathrm{~d}$ & $102,05 \mathrm{a}$ \\
\hline Tx644 B & $3,29 d$ & NR & $0,22 \mathrm{~d}$ & $122,99 b$ & $27,67 b$ & & $1,90 \mathrm{c}$ & I & $0,67 \mathrm{a}$ & $219,52 \mathrm{c}$ & $146,46 a$ \\
\hline Tx645 B & $1,75 \mathrm{f}$ & NR & $0,25 \mathrm{~d}$ & $59,66 \mathrm{~d}$ & $15,00 \mathrm{~b}$ & & $0,81 \mathrm{~d}$ & I & $0,40 \mathrm{a}$ & $156,31 \mathrm{~d}$ & $63,11 \mathrm{a}$ \\
\hline \multirow[t]{2}{*}{ TX7078R } & $3,66 \mathrm{c}$ & $\mathrm{R}$ & $0,47 \mathrm{~b}$ & $66,29 d$ & $31,21 \mathrm{~b}$ & & $1,92 \mathrm{c}$ & I & $0,59 \mathrm{a}$ & $252,09 \mathrm{c}$ & $147,53 \mathrm{a}$ \\
\hline & \multicolumn{11}{|c|}{ Média } \\
\hline Geral & 3,40 & - & 0,33 & 97,75 & 28,77 & Geral & 2,03 & - & 0,92 & 200,30 & 154,86 \\
\hline Responsivo & 4,37 & - & 0,38 & 114,63 & 36,76 & Ineficiente & 1,72 & - & 0,87 & 193,13 & 130,70 \\
\hline Não responsivo & 2,53 & - & 0,28 & 82,65 & 21,61 & Eficiente & 3,14 & - & 1,12 & 225,41 & 239,39 \\
\hline
\end{tabular}

${ }^{(1)} \mathrm{R}$, responsivas; NR, não responsivas; I, ineficientes; e E, eficientes. ${ }^{(2)}$ Médias seguidas de letras iguais não diferem pelo teste de Scott \& Knott, a $5 \%$ de probabilidade. 
Tabela 4. Produtividade relativa $\left(\mathrm{P}_{\mathrm{REL}}\right)$ e classificação ${ }^{(1)}$ de linhagens com base na produtividade, e eficiências de recuperação aparente $\left(\mathrm{E}_{\mathrm{REC}}\right)$, fisiológica ( $\left.\mathrm{E}_{\mathrm{FISIO}}\right)$ e agronômica $\left(\mathrm{E}_{\mathrm{AGRO}}\right)$ em dois níveis de adubação fosfatada, em sorgo (Sorghum bicolor) granífero ${ }^{(2)}$.

\begin{tabular}{|c|c|c|c|c|c|}
\hline \multirow[t]{2}{*}{ Linhagem } & \multirow[t]{2}{*}{ Classe } & \multirow{2}{*}{$\begin{array}{c}\mathrm{P}_{\mathrm{REL}} \\
(\%)\end{array}$} & $\mathrm{E}_{\mathrm{REC}}$ & $\mathrm{E}_{\text {FISIO }}$ & $\mathrm{E}_{\mathrm{AGRO}}$ \\
\hline & & & \multicolumn{3}{|c|}{ 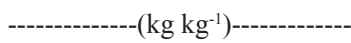 } \\
\hline ARG1 & IR & $63,6 \mathrm{a}$ & $0,26 \mathrm{~b}$ & $1,83 \mathrm{a}$ & $23,97 \mathrm{c}$ \\
\hline ATF13B & INR & $60,9 \mathrm{a}$ & $0,25 b$ & $1,76 \mathrm{a}$ & $16,11 \mathrm{~d}$ \\
\hline ATF14B & INR & $35,2 \mathrm{c}$ & $0,27 b$ & $1,36 \mathrm{a}$ & $8,71 \mathrm{e}$ \\
\hline ATF40B & ER & $40,1 \mathrm{c}$ & $0,30 \mathrm{~b}$ & $1,81 \mathrm{a}$ & $14,16 \mathrm{~d}$ \\
\hline ATF53B & INR & $68,3 \mathrm{a}$ & $0,25 \mathrm{~b}$ & $1,85 \mathrm{a}$ & $21,18 \mathrm{c}$ \\
\hline ATF54 (f186) & IR & $52,8 \mathrm{~b}$ & $0,15 \mathrm{c}$ & $1,59 \mathrm{a}$ & $23,28 \mathrm{c}$ \\
\hline ATF54 (f206) & IR & $50,0 \mathrm{~b}$ & $0,20 \mathrm{c}$ & $1,73 \mathrm{a}$ & $21,01 \mathrm{c}$ \\
\hline ATF54 (f240) & IR & $72,9 \mathrm{a}$ & $0,08 \mathrm{c}$ & $1,59 \mathrm{a}$ & $35,28 b$ \\
\hline ATF54 (f596) & IR & $42,8 \mathrm{c}$ & $0,17 \mathrm{c}$ & $0,86 \mathrm{c}$ & $18,31 \mathrm{~d}$ \\
\hline ATF54 (f61) & IR & $38,4 \mathrm{c}$ & $0,21 \mathrm{c}$ & $1,67 \mathrm{a}$ & $14,34 d$ \\
\hline ATF54 (f64) & IR & $54,8 \mathrm{~b}$ & $0,28 \mathrm{~b}$ & $1,55 \mathrm{a}$ & $21,55 \mathrm{c}$ \\
\hline ATF54B & INR & $36,8 \mathrm{c}$ & $0,22 b$ & $1,66 \mathrm{a}$ & $15,33 \mathrm{~d}$ \\
\hline BR005R & ENR & $0,2 \mathrm{~d}$ & $0,16 \mathrm{c}$ & $2,19 \mathrm{a}$ & $11,55 \mathrm{~d}$ \\
\hline BR007B & IR & $65,1 \mathrm{a}$ & $0,38 b$ & $1,87 \mathrm{a}$ & $37,99 \mathrm{~b}$ \\
\hline BR008B & IR & $65,2 \mathrm{a}$ & $0,44 \mathrm{a}$ & $1,72 \mathrm{a}$ & $38,59 \mathrm{~b}$ \\
\hline BR012R & INR & $20,8 \mathrm{c}$ & $0,23 b$ & $0,76 \mathrm{c}$ & $5,25 \mathrm{e}$ \\
\hline BR012 (1B) & ENR & $0,1 \mathrm{~d}$ & $0,36 \mathrm{~b}$ & $1,49 \mathrm{a}$ & $0,06 \mathrm{e}$ \\
\hline BR012 (2B) & INR & $11,6 \mathrm{~d}$ & $0,27 \mathrm{~b}$ & $1,71 \mathrm{a}$ & $3,21 \mathrm{e}$ \\
\hline BR012 (R6) & ENR & $0,4 \mathrm{~d}$ & $0,30 \mathrm{~b}$ & $1,52 \mathrm{a}$ & $0,90 \mathrm{e}$ \\
\hline ВTX623 & INR & $33,9 \mathrm{c}$ & $0,22 b$ & $1,23 b$ & $8,90 \mathrm{e}$ \\
\hline CMSXS110 & INR & $33,4 \mathrm{c}$ & $0,29 b$ & $1,20 \mathrm{~b}$ & $11,23 d$ \\
\hline CMSXS136 & INR & $33,2 \mathrm{c}$ & $0,13 \mathrm{c}$ & $0,57 \mathrm{c}$ & $4,39 \mathrm{e}$ \\
\hline CMSXS180R & ER & $28,6 \mathrm{c}$ & $0,23 b$ & $1,25 \mathrm{c}$ & $11,92 d$ \\
\hline CMSXS225 & ENR & $3,5 \mathrm{~d}$ & $0,33 b$ & $1,99 \mathrm{a}$ & $1,14 \mathrm{e}$ \\
\hline CMSXS226 & INR & $31,5 \mathrm{c}$ & $0,18 \mathrm{c}$ & $1,33 \mathrm{a}$ & $10,62 d$ \\
\hline CMSXS227 & INR & $23,2 \mathrm{c}$ & $0,16 \mathrm{c}$ & $0,99 \mathrm{~b}$ & $4,57 \mathrm{e}$ \\
\hline IS3620C & INR & $33,4 \mathrm{c}$ & $0,16 \mathrm{c}$ & $1,38 \mathrm{a}$ & $7,49 \mathrm{e}$ \\
\hline P9401 & IR & $78,4 \mathrm{a}$ & $0,47 \mathrm{a}$ & $1,91 \mathrm{a}$ & $45,40 \mathrm{a}$ \\
\hline P9405 & ENR & $21,4 \mathrm{c}$ & $0,45 \mathrm{a}$ & $0,32 \mathrm{c}$ & $7,87 \mathrm{e}$ \\
\hline QL3 & IR & $52,6 \mathrm{~b}$ & $0,35 b$ & $1,62 \mathrm{a}$ & $25,43 \mathrm{c}$ \\
\hline SC414-12E & IR & $60,2 \mathrm{a}$ & $0,28 \mathrm{~b}$ & $1,86 \mathrm{a}$ & $30,52 b$ \\
\hline SC566 & ER & $40,0 \mathrm{c}$ & $0,25 b$ & $1,64 \mathrm{a}$ & $23,45 \mathrm{c}$ \\
\hline Tx643 B & INR & $7,7 \mathrm{~d}$ & $0,09 \mathrm{c}$ & $2,05 \mathrm{a}$ & $1,26 \mathrm{e}$ \\
\hline Tx644 B & INR & $42,1 \mathrm{c}$ & $0,14 \mathrm{c}$ & $1,69 \mathrm{a}$ & $14,89 \mathrm{~d}$ \\
\hline Tx645 B & INR & $53,6 \mathrm{~b}$ & $0,23 b$ & $1,50 \mathrm{a}$ & $10,11 \mathrm{~d}$ \\
\hline \multirow[t]{2}{*}{ TX7078R } & IR & $47,5 \mathrm{~b}$ & $0,46 \mathrm{a}$ & $1,46 a$ & $18,68 \mathrm{~d}$ \\
\hline & \multicolumn{5}{|c|}{ Média } \\
\hline Geral & & 39,01 & 0,26 & 1,51 & 15,80 \\
\hline ER & & 32,53 & 0,31 & 1,26 & 14,35 \\
\hline ENR & & 1,05 & 0,29 & 1,80 & 3,41 \\
\hline IR & & 57,25 & 0,29 & 1,64 & 27,26 \\
\hline INR & & 35,04 & 0,21 & 1,40 & 9,55 \\
\hline
\end{tabular}

(1)IR, ineficientes e responsivas; INR, ineficientes e não responsivas; ER, eficientes e responsivas; e ENR, eficientes e não responsivas. ${ }^{(2)}$ Médias seguidas de letras iguais não diferem pelo teste de Scott \& Knott, a 5\% de probabilidade. aestivum L.) por Ozturk et al. (2005), milho por Li et al. (2008) e sorgo por Rocha et al. (2010). De Campos et al. (2013) também observaram essa variação em espécies nativas da Austrália. Por meio de análises proteômicas, Li et al. (2008) demonstraram que a secreção de citrato, o metabolismo de açúcares e a proliferação de células da raiz são as principais razões para a maior tolerância a solos deficientes em $\mathrm{P}$, no milho.

Quanto à responsividade das linhagens ao $\mathrm{P}$, observou-se que oito foram agrupadas no grupo a, com maior $\mathrm{P}_{\mathrm{REL}}$ (Tabela 4). Essas linhagens podem contribuir na geração de híbridos mais responsivos, por meio do aumento do aproveitamento do adubo fosfatado aplicado ao solo. Para o caráter $E_{R E C}$, as linhagens P9401, TX7078R, P9405 e BR008B apresentaram maior recuperação de $\mathrm{P}$ (grupo a). Entretanto, a diferença entre os grupos responsivos (R) e não responsivos (NR) foi de apenas $0,07 \mathrm{~kg} \mathrm{~kg}^{-1}$. Para $\mathrm{E}_{\mathrm{FISIO}}$, somente oito linhagens foram agrupadas entre as de pior desempenho (grupos b e c), e o caráter variou de $0,32 \mathrm{~kg} \mathrm{~kg}^{-1}$ (P9405) a 2,19 $\mathrm{kg} \mathrm{kg}^{-1}$ (BR005R). Isso indica que a linhagem BR005R produziu, em média, $219 \mathrm{~kg}$ a mais de matéria seca de grãos, para cada quilograma de $\mathrm{P}$, que as plantas no tratamento controle sob baixa disponibilidade de P. Segundo Lynch (2011), o custo metabólico da maior exploração do solo pelas raízes é componente importante no crescimento de plantas sob baixa disponibilidade de P. Os autores constataram que, fisiologicamente, a planta que investe em mais raízes pode acabar produzindo uma grande quantidade de tecido inativo, o que promoveria um gasto energético interno desnecessário e diminuiria sua produtividade.

A linhagem P9401 apresentou a maior $E_{\text {AGRO, }}$ com alto desempenho em condições de adubação adequada e desempenho bem inferior sob deficiência de $\mathrm{P}$ (Tabela 4). No caso dessa linhagem, a maior $\mathrm{E}_{\mathrm{AgRO}}$ deveu-se, principalmente, à baixa produtividade sob baixa disponibilidade de $\mathrm{P}$, tendo-se produzido $45 \mathrm{~kg}$ de grãos para cada quilograma de $\mathrm{P}$ disponível a mais no tratamento controle, em comparação ao tratamento sem adubação fosfatada.

Dezesseis linhagens foram indicadas como adaptadas a condições de disponibilidade adequada de $\mathrm{P}$, de acordo com a soma de postos dos caracteres: produtividade, $E_{\mathrm{ABS}}, \mathrm{E}_{\mathrm{UTIL}}$ e $\mathrm{E}_{\mathrm{USO}}$, estimados no tratamento controle; e $\mathrm{P}_{\mathrm{REL}}, \mathrm{E}_{\mathrm{REC}}, \mathrm{E}_{\mathrm{FISIO}}$ e $\mathrm{E}_{\mathrm{AGRO}}$, estimados por equações com os dois níveis de disponibilidade de P. Essas linhagens 
Tabela 5. Ranques das linhagens eficientes e responsivas de sorgo (Sorghum bicolor) com as respectivas classificações, com base na soma de postos dos caracteres avaliados.

\begin{tabular}{|c|c|c|c|c|c|c|c|c|c|c|c|c|c|c|c|c|c|}
\hline \multirow[t]{2}{*}{ Linhagem } & \multicolumn{10}{|c|}{ Responsividade ao $\mathrm{P}^{(1)}$} & \multicolumn{7}{|c|}{ Eficiência ao $\mathrm{P}^{(2)}$} \\
\hline & Prod & $\mathrm{E}_{\mathrm{ABS}}$ & $\mathrm{E}_{\text {UTIL }}$ & $\mathrm{E}_{\mathrm{USO}}$ & $\mathrm{P}_{\mathrm{REL}}$ & $\mathrm{E}_{\mathrm{REC}}$ & $\mathrm{E}_{\mathrm{FISIO}}$ & $\mathrm{E}_{\mathrm{AGRO}}$ & Soma & $\mathrm{Cl}$ & Prod & $\mathrm{E}_{\mathrm{ABS}}$ & $\mathrm{E}_{\text {UTIL }}$ & $\mathrm{E}_{\text {Uso }}$ & Soma & $\mathrm{Cl}$ & $\mathrm{Cf}$ \\
\hline ARG1 & 15 & 21 & 11 & 15 & 6 & 16 & 8 & 7 & 99 & $\mathrm{R}$ & 31 & 29 & 15 & 31 & 106 & I & IR \\
\hline ATF13B & 27 & 29 & 19 & 27 & 7 & 18 & 10 & 15 & 152 & NR & 34 & 35 & 2 & 34 & 105 & I & INR \\
\hline ATF14B & 31 & 24 & 27 & 31 & 21 & 14 & 27 & 26 & 201 & NR & 27 & 32 & 5 & 27 & 91 & I & INR \\
\hline ATF40B & 5 & 1 & 24 & 5 & 17 & 9 & 9 & 19 & 89 & $\mathrm{R}$ & 2 & 3 & 16 & 2 & 23 & $\mathrm{E}$ & ER \\
\hline ATF53B & 25 & 27 & 14 & 25 & 3 & 17 & 7 & 11 & 129 & $\mathrm{R}$ & 35 & 34 & 7 & 35 & 111 & I & IR \\
\hline ATF54 (f186) & 9 & 16 & 12 & 9 & 11 & 32 & 19 & 9 & 117 & $\mathrm{R}$ & 18 & 2 & 35 & 22 & 77 & I & IR \\
\hline ATF54 (f206) & 11 & 20 & 7 & 11 & 13 & 26 & 11 & 12 & 111 & $\mathrm{R}$ & 17 & 13 & 28 & 17 & 75 & I & IR \\
\hline ATF54 (f240) & 7 & 36 & 1 & 8 & 2 & 36 & 20 & 4 & 114 & $\mathrm{R}$ & 32 & 36 & 1 & 32 & 101 & I & IR \\
\hline ATF54 (f596) & 10 & 7 & 23 & 10 & 15 & 28 & 33 & 14 & 140 & $\mathrm{R}$ & 10 & 1 & 36 & 9 & 56 & $\mathrm{E}$ & ER \\
\hline ATF54 (f61) & 16 & 28 & 5 & 16 & 19 & 25 & 15 & 18 & 142 & $\mathrm{R}$ & 13 & 30 & 4 & 11 & 58 & $\mathrm{E}$ & ER \\
\hline ATF54 (f64) & 13 & 11 & 22 & 14 & 9 & 13 & 21 & 10 & 113 & $\mathrm{R}$ & 25 & 6 & 34 & 25 & 90 & I & IR \\
\hline ATF54B & 23 & 34 & 2 & 23 & 20 & 24 & 16 & 16 & 158 & NR & 15 & 11 & 26 & 12 & 64 & E & ENR \\
\hline BR005R & 24 & 31 & 9 & 24 & 35 & 31 & 1 & 21 & 176 & NR & 1 & 22 & 3 & 1 & 27 & $\mathrm{E}$ & ENR \\
\hline BR007B & 3 & 9 & 8 & 3 & 5 & 5 & 5 & 3 & 41 & $\mathrm{R}$ & 21 & 17 & 22 & 20 & 80 & I & IR \\
\hline BR008B & 1 & 2 & 17 & 1 & 4 & 4 & 12 & 2 & 43 & $\mathrm{R}$ & 19 & 9 & 32 & 18 & 78 & I & IR \\
\hline BR012R & 30 & 19 & 29 & 30 & 30 & 22 & 34 & 29 & 223 & NR & 24 & 20 & 27 & 24 & 95 & I & INR \\
\hline BR012 (1B) & 34 & 6 & 36 & 34 & 36 & 6 & 24 & 36 & 212 & NR & 6 & 5 & 20 & 6 & 37 & E & ENR \\
\hline BR012 (2B) & 26 & 18 & 31 & 26 & 31 & 15 & 13 & 32 & 192 & NR & 11 & 18 & 14 & 10 & 53 & $\mathrm{E}$ & ENR \\
\hline BR012 (R6) & 29 & 14 & 34 & 29 & 34 & 10 & 22 & 35 & 207 & NR & 5 & 21 & 6 & 4 & 36 & $\mathrm{E}$ & ENR \\
\hline BTX623 & 28 & 22 & 26 & 28 & 22 & 23 & 30 & 25 & 204 & NR & 26 & 19 & 29 & 26 & 100 & I & INR \\
\hline CMSXS110 & 21 & 12 & 25 & 20 & 23 & 11 & 31 & 22 & 165 & NR & 16 & 15 & 21 & 15 & 67 & $\mathrm{E}$ & ENR \\
\hline CMSXS136 & 18 & 25 & 10 & 18 & 25 & 34 & 35 & 31 & 196 & NR & 9 & 4 & 33 & 13 & 59 & E & ENR \\
\hline CMSXS180R & 12 & 17 & 16 & 13 & 27 & 21 & 29 & 20 & 155 & NR & 7 & 8 & 19 & 7 & 41 & $\mathrm{E}$ & ENR \\
\hline CMSXS225 & 22 & 10 & 32 & 22 & 33 & 8 & 3 & 34 & 164 & NR & 4 & 16 & 9 & 5 & 34 & $\mathrm{E}$ & ENR \\
\hline CMSXS226 & 20 & 23 & 15 & 21 & 26 & 27 & 28 & 23 & 183 & NR & 12 & 10 & 31 & 16 & 69 & $\mathrm{E}$ & ENR \\
\hline CMSXS227 & 33 & 30 & 28 & 33 & 28 & 30 & 32 & 30 & 244 & NR & 28 & 24 & 30 & 29 & 111 & I & INR \\
\hline IS3620C & 32 & 33 & 21 & 32 & 24 & 29 & 26 & 28 & 225 & NR & 30 & 27 & 18 & 28 & 103 & I & INR \\
\hline P9401 & 4 & 5 & 13 & 4 & 1 & 1 & 4 & 1 & 33 & $\mathrm{R}$ & 33 & 31 & 11 & 33 & 108 & I & IR \\
\hline P9405 & 17 & 3 & 35 & 17 & 29 & 3 & 36 & 27 & 167 & NR & 8 & 14 & 12 & 8 & 42 & $\mathrm{E}$ & ENR \\
\hline QL3 & 8 & 8 & 18 & 7 & 12 & 7 & 18 & 6 & 84 & $\mathrm{R}$ & 14 & 12 & 24 & 14 & 64 & $\mathrm{E}$ & ER \\
\hline SC414-12E & 6 & 15 & 6 & 6 & 8 & 12 & 6 & 5 & 64 & $\mathrm{R}$ & 23 & 23 & 17 & 23 & 86 & I & IR \\
\hline SC566 & 2 & 13 & 3 & 2 & 18 & 19 & 17 & 8 & 82 & $\mathrm{R}$ & 3 & 7 & 13 & 3 & 26 & $\mathrm{E}$ & ER \\
\hline Tx643 B & 36 & 35 & 20 & 36 & 32 & 35 & 2 & 33 & 229 & NR & 29 & 25 & 25 & 30 & 109 & I & INR \\
\hline Tx644 B & 19 & 32 & 4 & 19 & 16 & 33 & 14 & 17 & 154 & NR & 22 & 26 & 10 & 21 & 79 & I & INR \\
\hline Tx645 B & 35 & 26 & 33 & 35 & 10 & 20 & 23 & 24 & 206 & NR & 36 & 33 & 23 & 36 & 128 & I & INR \\
\hline TX7078R & 14 & 4 & 30 & 12 & 14 & 2 & 25 & 13 & 114 & $\mathrm{R}$ & 20 & 28 & 8 & 19 & 75 & I & IR \\
\hline
\end{tabular}

${ }^{(1)}$ Responsividade avaliada em condições adequadas de adubação fosfatada e de acordo com a relação entre as condições controle e estresse. ${ }^{(2)}$ Eficiência avaliada em condições de estresse. Prod, produtividade; $\mathrm{E}_{\mathrm{ABS}}$, eficiência de absorção; $\mathrm{E}_{\mathrm{UTIL}}$, eficiência de utilização; $\mathrm{E}_{\mathrm{USo}}$, eficiência de uso; $\mathrm{P}_{\mathrm{REL}}$, produtividade relativa; $\mathrm{E}_{\mathrm{REC}}$, eficiência de recuperação; $\mathrm{E}_{\mathrm{FISIO}}$, eficiência fisiológica; $\mathrm{E}_{\mathrm{AGRO}}$, eficiência agronômica. Classificações (Cl, parcial; e Cf, final): $\mathrm{R}$, responsivas; NR, não responsivas; I, ineficientes; E, eficientes; IR, ineficientes e responsivas; INR, ineficientes e não responsivas; ER, eficientes e responsivas; ENR, eficientes e não responsivas. 
apresentaram pontuações entre 33 e 142 no ranque (Tabela 5). Para a eficiência produtiva, foram avaliados os caracteres produtividade, $\mathrm{E}_{\mathrm{ABS}}, \mathrm{E}_{\mathrm{UTIL}}$ e $\mathrm{E}_{\mathrm{USO}}$, em condições de estresse, e também foram classificadas 16 linhagens, pela soma de postos, com pontuações entre 23 e 69 no ranque. As seguintes linhagens apresentaram, de forma simultânea, alta responsividade e alta eficiência produtiva em relação ao P: ATF40B, ATF54 (f61), ATF54 (f596), QL3 e SC566. Essas linhagens, portanto, devem ser priorizadas para a formação de híbridos que podem ser destinados a diferentes nichos de mercado, que demandem tanto o emprego de alta quanto de baixa tecnologia.

Cabe destacar que a classificação com uso da produtividade apenas, apesar de simples, foi eficiente em classificar as linhagens como responsivas, tendo apresentado $92 \%$ de coincidência com a classificação obtida com a soma de postos de Mulamba \& Mock (1978), na avaliação com níveis adequados de $\mathrm{P}$ (Tabela 5). Quanto à eficiência produtiva em relação ao $\mathrm{P}$, a classificação de acordo com a produtividade apresentou $78 \%$ de coincidência com a classificação pela soma de postos. Deve-se ressaltar, porém, que o uso da produtividade como único caráter identificador de linhagens eficientes e responsivas ao fósforo, embora adequado, deve restringir-se às fases iniciais do programa de melhoramento, quando se avalia elevado número de linhagens. Por fim, é importante mencionar a pertinência da avaliação de doses intermediárias de $\mathrm{P}$, que poderiam promover alterações metabólicas benéficas à produtividade nas plantas.

\section{Conclusões}

1. Há variabilidade entre as linhagens de sorgo (Sorghum bicolor) granífero quanto à eficiência produtiva e à responsividade ao fósforo, o que aponta para a possibilidade de obtenção de híbridos para diferentes nichos de mercado.

2. A classificação das linhagens de acordo apenas com a produtividade é capaz de identificar e discriminar linhagens eficientes e responsivas ao fósforo, em sorgo granífero.

\section{Agradecimentos}

À Fundação de Amparo à Pesquisa do Estado de Minas Gerais (Fapemig), pela bolsa de apoio técnico; e à Fundação McKnight, pelo apoio financeiro.

\section{Referências}

CRUZ, C.D. Programa Genes: biometria. Viçosa: Ed. da UFV, 2006. 382p.

DE CAMPOS, M.C.R.; PEARSE, S.J.; OLIVEIRA, R.S.; LAMBERS, H. Downregulation of net phosphorus-uptake capacity is inversely related to leaf phosphorus-resorption proficiency in four species from a phosphorus-impoverished environment. Annals of Botany, v.111, p.445-454, 2013. DOI: 10.1093/aob/mcs299.

DOVALE, J.C.; MAIA, C.; FRITSCHE-NETO, R.; MIRANDA, G.V.; CAVATTE, P.C. Genetic responses of traits relationship to components of nitrogen and phosphorus use efficiency in maize. Acta Scientiarum. Agronomy, v.35, p.31-38, 2013. DOI: 10.4025/ actasciagron.v35i1.15237.

FRITSCHE-NETO, R.; MIRANDA, G.V.; DELIMA, R.O.; SOUZA, L.V.D.; SILVA, J.D. Inheritance of traits associated with phosphorus utilization efficiency in maize. Pesquisa Agropecuária Brasileira, v.45, p.465-471, 2010. DOI: 10.1590/ S0100-204X2010000500005.

GODFRAY, H.C.J.; BEDDINGTON, J.R.; CRUTE, I.R.; HADDAD, L.; LAWRENCE, D.; MUIR, J.F.; PRETTY, J.; ROBINSON, S.; THOMAS, S.M.; TOULMIN, C. Food security: the challenge of feeding 9 billion people. Science, v.327, p.812-818, 2010. DOI: 10.1126/science.1185383.

GOOD, A.G.; SHRAWAT, A.K.; MUENCH, D.G. Can less yield more? Is reducing nutrient input into the environment compatible with maintaining crop production? Trends in Plant Science, v.9, p.597-605, 2004. DOI: 10.1016/j.tplants.2004.10.008.

LI, K.; XU, C.; LI, Z.; ZHANG, K.; YANG, A.; ZHANG, J. Comparative proteome analyses of phosphorus responses in maize (Zea mays L.) roots of wild-type and a low-P-tolerant mutant reveal root characteristics associated with phosphorus efficiency. Plant Journal, v.55, p.927-939, 2008. DOI: 10.1111/j.1365-313X .2008.03561.x.

LUO, L.; WANG, X.C.; GUO, W.; NGO, H.H.; CHEN, Z. Impact assessment of excess discharges of organics and nutrients into aquatic systems by thermodynamic entropy calculation. Journal of Environmental Management, v.112, p.45-52, 2012. DOI: 10.1016/j.jenvman.2012.07.011.

LYNCH, J.P. Root phenes for enhanced soil exploration and phosphorus acquisition: tools for future crops. Plant Physiology, v.156, p.1041-1049, 2011. DOI: 10.1104/pp.111.175414.

MALINGREAU, J.-P.; EVA, H.; MAGGIO, A. NPK: will there be enough plant nutrients to feed a world of 9 billion in 2050 ? Luxembourg: European Commission, 2012. 30p. (Foresight and horizon scanning series). DOI: 10.2788/26603.

MCGILL, S.M. 'Peak' phosphorus? The implications of phosphate scarcity for sustainable investors. Journal of Sustainable Finance and Investment, v.2, p.222-239, 2012. DOI: 10.1080/20430795.2012.742635.

MULAMBA, N.-N.; MOCK, J.J. Improvement of yield potential of the Eto Blanco maize (Zea mays L.) population by breeding for plant traits. Egyptian Journal of Genetics and Cytology, v.7, p.40-51, 1978. 
OLIVEIRA, T.C. de; SILVA, J.; SALGADO, F.H.M.; SOUSA, S.A.; FIDELIS, R.R. Eficiência e resposta à aplicação de fósforo em feijão comum em solos de cerrado. Revista Verde de Agroecologia e Desenvolvimento Sustentável, v.7, p.16-24, 2012.

OZTURK, L.; EKER, S.; TORUN, B.; CAKMAK, I. Variation in phosphorus efficiency among 73 bread and durum wheat genotypes grown in a phosphorus-deficient calcareous soil. Plant and Soil, v.269, p.69-80, 2005. DOI: 10.1007/s11104-004-0469-z.

PARENTONI, S.N. Estimativa de efeitos gênicos de diversos caracteres relacionados à eficiência e resposta ao fósforo em milho tropical. 2008. 207p. Tese (Doutorado) - Escola Superior de Agricultura Luiz de Queiroz, Piracicaba.

RAMAEKERS, L.; REMANS, R.; RAO, I.M.; BLAIR, M.W.; VANDERLEYDEN, J. Strategies for improving phosphorus acquisition efficiency of crop plants. Field Crops Research, v.117, p.169-176, 2010. DOI: 10.1016/j.fcr.2010.03.001.

RANNO, S.K.; SILVA, L.S. da; GATIBONI, L.C.; RHODEN, A.C. Capacidade de adsorção de fósforo em solos de várzea do Estado do Rio Grande do Sul. Revista Brasileira de Ciência do Solo, v.31, p.21-28, 2007. DOI: 10.1590/S0100-06832007000100003.

ROCHA, M.C. da; MIRANDA, G.V.; VASCONCELOS, M.J.V.; MAGALHÃES, P.C.; CARVALHO JÚNIOR, G.A. de; SILVA, L.A.; SOARES, M.O.; CANTÃO, F.R.O.; RODRIGUES, F.; SCHAFFERT, R.E. Caracterização da morfologia radicular de genótipos contrastantes de sorgo em baixos e altos níveis de fósforo. Revista Brasileira de Milho e Sorgo, v.9, p.65-78, 2010.

SANTOS, D.R. dos; GATIBONI, L.C.; KAMINSKI, J. Fatores que afetam a disponibilidade do fósforo e o manejo da adubação fosfatada em solos sob sistema plantio direto. Ciência Rural, v.38, p.576-586, 2008. DOI: 10.1590/S0103-84782008000200049.

SCHAFFERT, R.E.; ALVES, V.M.C.; PITTA, G.V.E.; BAHIA, A.F.C.; SANTOS, F.G. Genetic variability in sorghum for $\mathrm{P}$ efficiency and responsiveness. Plant Nutrition, v.92, p.72-73, 2001. DOI: 10.1007/0-306-47624-X_34.

SISTEMA brasileiro de classificação de solos. Brasília: Embrapa Produção de Informação; Rio de Janeiro: Embrapa Solos, 1999. 412p.

$\overline{\text { Recebido em } 11 \text { de fevereiro de } 2014 \text { e aprovado em } 5 \text { de agosto de } 2014}$

Pesq. agropec. bras., Brasília, v.49, n.8, p.613-621, ago. 2014 DOI: $10.1590 / \mathrm{S} 0100-204 \mathrm{X} 2014000800005$ 